\title{
Ultrabroadband terahertz spectroscopy of chalcogenide glasses
}

Zalkovskij, Maksim; Bisgaard, Christer Zoffmann; Novitsky, Andrey; Malureanu, Radu; Savastru, Dan; Popescu, Aurelian; Jepsen, Peter Uhd; Lavrinenko, Andrei

Published in:

Applied Physics Letters

Link to article, DOI:

$10.1063 / 1.3676443$

Publication date:

2012

Document Version

Publisher's PDF, also known as Version of record

Link back to DTU Orbit

Citation (APA):

Zalkovskij, M., Bisgaard, C. Z., Novitsky, A., Malureanu, R., Savastru, D., Popescu, A., Jepsen, P. U., \& Lavrinenko, A. (2012). Ultrabroadband terahertz spectroscopy of chalcogenide glasses. Applied Physics Letters, 100(3), 031901. https://doi.org/10.1063/1.3676443

\section{General rights}

Copyright and moral rights for the publications made accessible in the public portal are retained by the authors and/or other copyright owners and it is a condition of accessing publications that users recognise and abide by the legal requirements associated with these rights.

- Users may download and print one copy of any publication from the public portal for the purpose of private study or research.

- You may not further distribute the material or use it for any profit-making activity or commercial gain

- You may freely distribute the URL identifying the publication in the public portal 


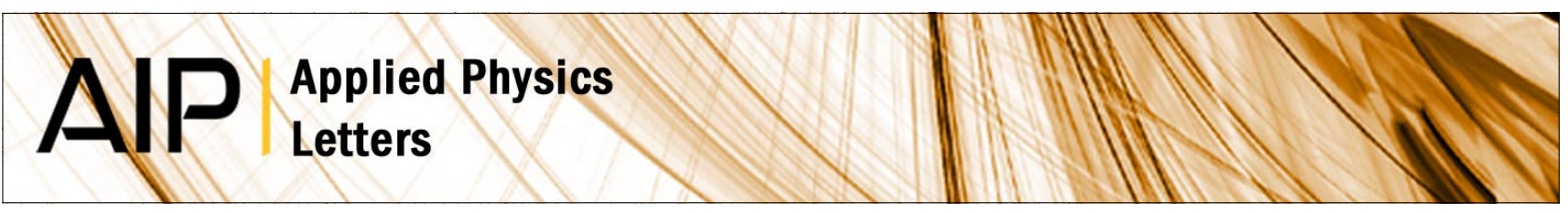

\section{Ultrabroadband terahertz spectroscopy of chalcogenide glasses}

Maksim Zalkovskij, Christer Zoffmann Bisgaard, Andrey Novitsky, Radu Malureanu, Dan Savastru et al.

Citation: Appl. Phys. Lett. 100, 031901 (2012); doi: 10.1063/1.3676443

View online: http://dx.doi.org/10.1063/1.3676443

View Table of Contents: http://apl.aip.org/resource/1/APPLAB/v100/i3

Published by the American Institute of Physics.

\section{Related Articles}

Analysis of Gouy phase shift for optimizing terahertz air-biased-coherent-detection

Appl. Phys. Lett. 100, 061105 (2012)

Solid effect dynamic nuclear polarization and polarization pathways

JCP: BioChem. Phys. 6, 01B602 (2012)

Solid effect dynamic nuclear polarization and polarization pathways

J. Chem. Phys. 136, 015101 (2012)

A compact SQUID-detected magnetic resonance imaging system under microtesla field in a magnetically unshielded environment

J. Appl. Phys. 110, 093903 (2011)

Design, construction, and testing of a large-cavity, 1-10 GHz Flygare-Balle spectrometer

Rev. Sci. Instrum. 82, 094103 (2011)

\section{Additional information on Appl. Phys. Lett.}

Journal Homepage: http://apl.aip.org/

Journal Information: http://apl.aip.org/about/about_the_journal

Top downloads: http://apl.aip.org/features/most_downloaded

Information for Authors: http://apl.aip.org/authors

\section{ADVERTISEMENT}

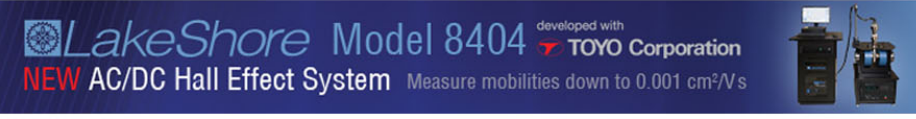




\title{
Ultrabroadband terahertz spectroscopy of chalcogenide glasses
}

\author{
Maksim Zalkovskij, ${ }^{1, a)}$ Christer Zoffmann Bisgaard, ${ }^{1}$ Andrey Novitsky, ${ }^{1}$ Radu Malureanu, ${ }^{1}$ \\ Dan Savastru, ${ }^{2}$ Aurelian Popescu, ${ }^{2}$ Peter Uhd Jepsen, ${ }^{1}$ and Andrei V. Lavrinenko ${ }^{1}$ \\ ${ }^{1}$ Department of Photonics Engineering, Technical University of Denmark, Ørsteds Plads 343, DK-2800 Kgs. \\ Lyngby, Denmark \\ ${ }^{2}$ National Institute of R\&D for Optoelectronics INOE 2000, Bucharest, Romania
}

(Received 3 November 2011; accepted 17 December 2011; published online 17 January 2012)

\begin{abstract}
Chalcogenide glasses are receiving a lot of attention due to their unique optical properties. In this paper we study the optical properties of $\mathrm{As}_{2} \mathrm{~S}_{3}$ and $\mathrm{GaLaS}$ glasses in a broad terahertz $(\mathrm{THz})$ frequency range $(0.2-18 \mathrm{THz})$. Complex dispersion behavior with drastic changes of refractive index and absorption coefficient is found for both glasses. We observe the breakdown of the universal power-law dependence of the absorption coefficient due to atomic vibrations observed at low $\mathrm{THz}$ frequencies in disordered materials, and see the transition to localized vibrational dynamics for the $\mathrm{As}_{2} \mathrm{~S}_{3}$ compound at higher frequencies. In addition, $\mathrm{As}_{2} \mathrm{~S}_{3}$ displays two transparency regions, at $7-8 \mathrm{THz}$ and $12.2 \mathrm{THz}$, of potential interest for future nonlinear applications in the THz range. (C) 2012 American Institute of Physics. [doi:10.1063/1.3676443]
\end{abstract}

Since the first optical characterization of chalcogenide glasses (ChGs) in the infrared region reported approximately six decades ago, ${ }^{1}$ a huge advancement has been made in implementing ChGs into optical devices. ${ }^{2}$ ChGs possess a unique combination of material properties including high refractive index, mid-infrared transparency, and high nonlinearities. ${ }^{3,4}$ The flexibility of ChGs towards micro- and nano-fabrication processing by means of nano-imprint lithography, ${ }^{5}$ etching, ${ }^{6}$ and direct laser writing of $2 \mathrm{D}$ photonic structure $^{7-9}$ together with their unique material parameters make ChGs promising candidates for numerous optical applications. ChGs have, for example, already been integrated into high-speed all-optical devices through four-wave mixing and cross phase modulation devices, ${ }^{10}$ optoelectrophoretic sensors for detection of microorganisms, ${ }^{11}$ and three-dimensional photonic crystals. ${ }^{7}$

Meanwhile, progress in methods for generating and detecting terahertz $(\mathrm{THz})$ radiation has spectacularly increased the interest of its applications in a variety of fields, e.g., in spectroscopy of organic tissues, ${ }^{12}$ chemical compounds, ${ }^{13}$ proteins,${ }^{14}$ and solid materials. ${ }^{15}$ There is strong demand on different THz-optic components. Some simple devices like dielectric mirrors, ${ }^{16}$ waveguides,${ }^{17}$ filters,${ }^{18}$ resonators, ${ }^{19}$ polarizer elements ${ }^{20,21}$ have been designed for $\mathrm{THz}$ frequencies. In the quest for innovative functional $\mathrm{THz}$ components, ChGs bear a great potential as the active material in nonlinear $\mathrm{THz}$ devices. However, at this stage it is essential to determine their optical properties at $\mathrm{THz}$ frequencies, which are mostly unknown.

In this study, we report on the optical parameters, represented by the refractive index and absorption coefficient, at terahertz frequencies for $\mathrm{As}_{2} \mathrm{~S}_{3}$ and GaLaS- two quite different chalcogenide glasses. At ambient temperature $\mathrm{As}_{2} \mathrm{~S}_{3}$ is a mechanically soft glass with low, less than $200^{\circ} \mathrm{C}$ glass transition temperature. GaLaS is mechanically hard, easily polished glass with a higher $580^{\circ} \mathrm{C}$ glass transition tempera-

$\overline{{ }^{a} \text { Electronic mail: mzal@fotonik.dtu.dk. }}$ ture. The former has become a widely used material for many optical applications due to its material parameters and flexibility towards fabrication. ${ }^{2,6,7,10}$ The GaLaS glass with the composition $70 \mathrm{Ga}_{2} \mathrm{~S}_{3}: 30 \mathrm{La}_{2} \mathrm{~S}_{3}$, on the other hand, is not so exploited yet. It is a material which shows high stability and resistance to mechanical and chemical wear thus making it an interesting material to be used for devices operating in harsh environments. The optical parameters were determined for frequencies spanning from 0.2 to $18 \mathrm{THz}$. Two different terahertz time-domain spectroscopy (THz-TDS) systems were utilized to cover such broad spectrum. At lower frequencies, ranging from 0.2 to $2.5 \mathrm{THz}$, we employ a

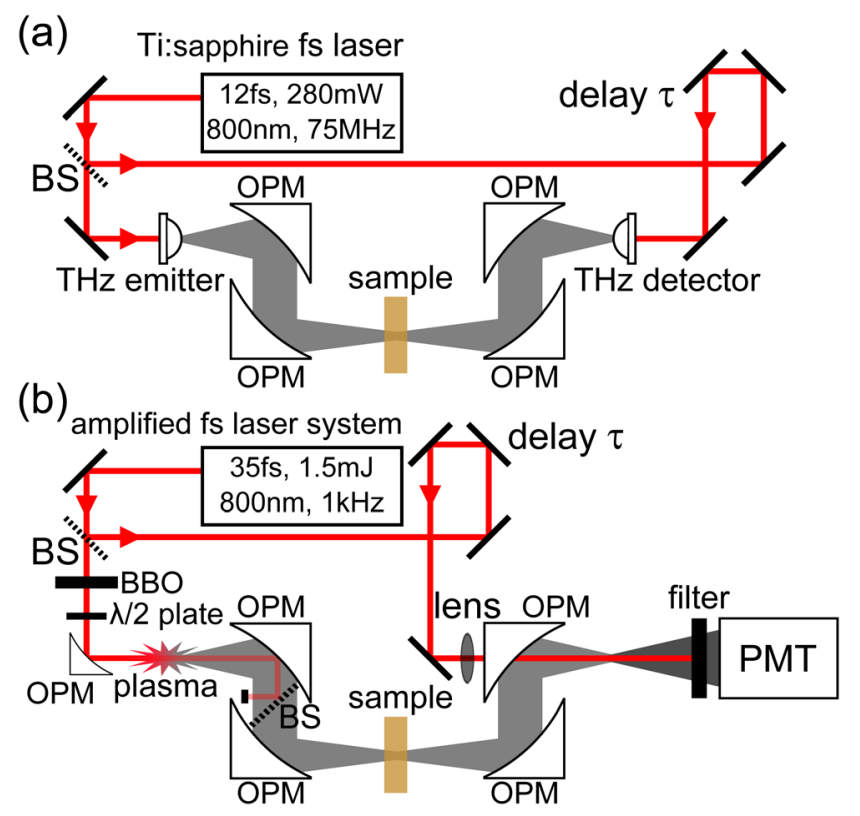

FIG. 1. (Color online) (a) Photoconductive (PC) THz-TDS setup. The terahertz pulse is emitted by a PC switch, which is able to generate terahertz radiation in a range of $0.2-2.5 \mathrm{THz}$ (b) $\mathrm{ABCD}$ THz-TDS setup. By focusing 35 fs laser pulse its second harmonic plasma can be generated, which produces a terahertz pulse with a broad frequency range 2-18 THz. OPM: offaxis parabolic mirror, $\mathrm{BS}$ : beam splitter, BBO: $\beta$-barium borate, PMT: photomultiplier tube. 
THz-TDS as illustrated in Fig. 1(a). ${ }^{22}$ The THz radiation is generated by femtosecond excitation of a low-temperaturegrown GaAs photoconductive switch (Menlo systems) with 800 -nm wavelength pulses from a femtosecond oscillator (Femto-Lasers Fusion Pro300). The detection of the terahertz pulses is performed by photoconductive sampling in a similar antenna, gated by the same Ti:sapphire laser. The photocurrent is recorded with a lock-in amplifier locked to the frequency $(13.2 \mathrm{kHz})$ of the modulation of the bias voltage on the THz emitter as function of the delay time $\tau$ and used to obtain the representation of the THz electric field $E_{T H z}(\tau)$.

The higher frequencies ranging from 2 to $18 \mathrm{THz}$ are generated by laser induced air plasma and detected in an air biased coherent detection (ABCD) setup. ${ }^{23,24}$ A schematic illustration of the THz-ABCD setup is shown in Fig. 1(b). To generate gas plasma a laser pulse (frequency $\omega$ ) of $35 \mathrm{fs,}$ $800 \mathrm{~nm}, 1.5 \mathrm{~mJ}$ with a repetition rate of $1 \mathrm{kHz}$ and its second harmonic $(2 \omega)$, generated in a $100 \mu \mathrm{m}$ thick $\beta$-barium borate (BBO) crystal, are focused collinearly by an off-axis paraboloidal mirror in a $\mathrm{N}_{2}$ purged chamber. The laser pulse is produced by a Ti:sapphire amplifier system (Spectra-Physics Spitfire). In the ABCD setup the $\mathrm{THz}$ field is detected by monitoring the THz-field-induced second harmonic $(2 \omega)$ of the $800-\mathrm{nm}$ probe beam, focused in the detection region to an intensity slightly below the plasma generation threshold. An AC bias field is applied to the interaction region to supply an optical bias to the second-harmonic generation, thus allowing field-resolved detection of the $\mathrm{THz}$ transient as function of the delay time $\tau$.

For the spectroscopic analysis, $\mathrm{As}_{2} \mathrm{~S}_{3}$ and GaLaS glasses were thermally deposited on $528 \mu \mathrm{m}$ thick, $2 \times 1 \mathrm{~cm}$ highresistivity silicon (HR Si) wafer pieces with resistivity of $10000 \Omega \mathrm{cm}$. The $\mathrm{As}_{2} \mathrm{~S}_{3}$ glasses were prepared by conventional melt-quenching techniques using arsenic and sulphur elements of high purity. The obtained bulk material was crumbled and put into a tantalum boat. The films were obtained by thermal evaporation technique in a vacuum of $5 \times 10^{-6}$ Torr. The electric current which heats the tantalum evaporator was adjusted to obtain film deposition rate of $2.5-3.0 \mathrm{~nm} / \mathrm{s}$. The films are asdeposited, without any heat treatment.

Lanthanum based glasses of composition $70 \% \mathrm{Ga}_{2} \mathrm{~S}_{3}$ and $30 \% \mathrm{La}_{2} \mathrm{~S}_{3}$ were prepared by a specially developed technique. The mixture of appropriate powdered components $\mathrm{Ga}_{2} \mathrm{~S}_{3}$ and $\mathrm{La}_{2} \mathrm{~S}_{3}$ is placed in a carbon glass crucible. The crucible is heated by radio-frequency induction at a frequency of $120 \mathrm{kHz}$. At high temperatures the melt becomes a conductor which contributes to material convection. Melting is done in a medium purged by argon gas. Other technological data and optical properties of glass are presented in Ref. 25 . Thin films are obtained from the $70 \% \mathrm{Ga}_{2} \mathrm{~S}_{3}, 30 \% \mathrm{La}_{2} \mathrm{~S}_{3}$ vitreous target using pulse laser deposition in vacuum $2 \times$ $10^{-6}$ Torr. The target is irradiated by $200 \mathrm{~mJ} / 10 \mathrm{~ns}$ pulses of the excimer laser with $248 \mathrm{~nm}$ wavelength. The silicon substrate is placed during ablation in an oven at a temperature of $100^{\circ} \mathrm{C}$, at a distance of $55 \mathrm{~mm}$ from the target.

One half of the HR Si wafer was covered with glass, whereas the uncovered area was used for the reference measurement. Three samples with different thicknesses were prepared for each glass: 13.6, 6.9, and $3.2 \mu \mathrm{m}$ for $\mathrm{As}_{2} \mathrm{~S}_{3}$ and 3.6, 2.3, and $1.3 \mu \mathrm{m}$ for GaLaS. Different thicknesses of the glass layer are used in order to control the precision of the restoration procedure. To extract material parameters from such thin glass samples one must take into account the FabryPérot (FP) etalon effect inside the glass layer. We follow the extraction procedure described by Duvillaret et $a .^{26}$ The complex refractive indices $\tilde{n}=n-i \kappa$ for the thin glass samples deposited on HR Si substrate is retrieved by inverting the amplitude transmission coefficient $\tilde{T}(\omega)$ expression

$$
\begin{aligned}
\tilde{T}(\omega)= & \frac{\tilde{E}_{\text {sam }}(\omega)}{\tilde{E}_{\text {ref }}(\omega)}=\frac{2 \tilde{n}\left(n_{S i}+n_{\text {air }}\right)}{\left(\tilde{n}+n_{S i}\right)\left(\tilde{n}+n_{\text {air }}\right)} \\
& \times \exp \left[-i\left(\tilde{n}-n_{\text {air }}\right) \frac{\omega \cdot d}{c}\right] \cdot \tilde{F} P, \\
\tilde{F} P= & \frac{1}{1-\frac{\left(\tilde{n}-n_{\text {air }}\right)\left(\tilde{n}-n_{S i}\right)}{\left(\tilde{n}+n_{\text {air }}\right)\left(\tilde{n}+n_{S i}\right)} \cdot \exp \left[-2 i(\tilde{n}) \frac{\omega \cdot d}{c}\right]} .
\end{aligned}
$$

In Eq. (1) $n_{S i}=3.2$ and $n_{\text {air }}=1$ are refractive indices of HR Si wafer and air respectively, $d$ is the thickness of the ChGs, $c$ is the speed of light in vacuum, and $\omega$ is the angular frequency. In addition to the thin ChGs samples, two additional free standing thick samples $\left(0.85 \mathrm{~mm} \mathrm{As}_{2} \mathrm{~S}_{3}\right.$ and $\left.1.40 \mathrm{~mm} \mathrm{GaLaS}\right)$ without substrate were characterized at low frequencies.

The thin samples were characterized by both THz-TDS systems in transmission configuration. The $0.85 \mathrm{~mm}$ thick $\mathrm{As}_{2} \mathrm{~S}_{3}$ and $1.40 \mathrm{~mm} \mathrm{GaLaS}$ were measured only at low frequencies because they were too absorptive for the broadband characterization with the THz-ABCD system. Figure 2 shows terahertz pulses after transmission through the reference (black trace) and through the $13.6 \mu \mathrm{m}$ thick $\mathrm{As}_{2} \mathrm{~S}_{3}$ sample (red trace) recorded in the time domain by the THz-ABCD setup.

As seen in Fig. 2 the terahertz pulse that propagates through the sample has lower amplitude, is delayed with respect to the reference pulse. Significant ringing is observed on the trailing edge of the pulse, indicative of a resonant response of the sample. The lower amplitude is due to absorption in the ChGs, while the additional delay is due to the longer optical path in ChGs when compared with air. The amplitude spectra in the frequency domain are then obtained by Fourier transformation of the time-domain data.

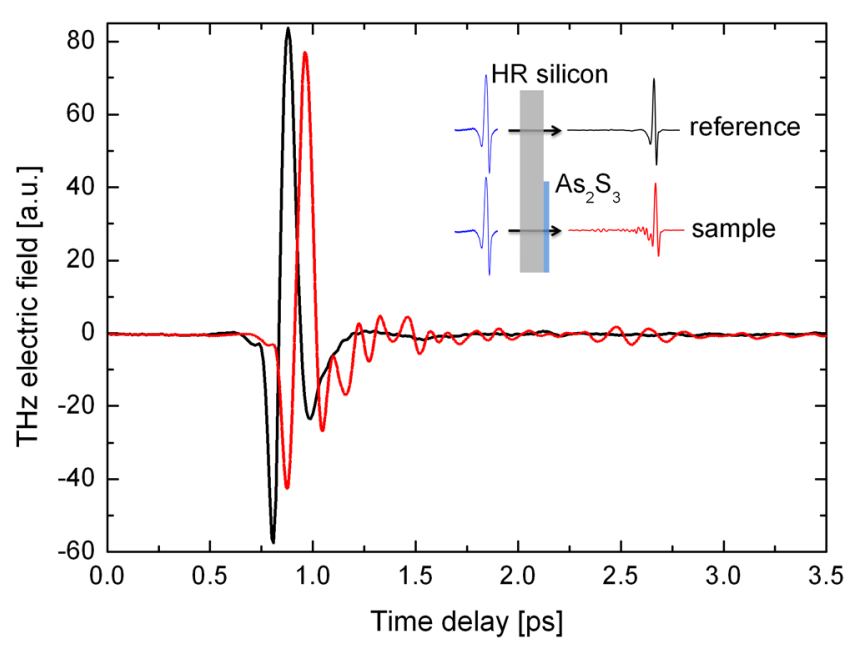

FIG. 2. (Color online) Measured terahertz pulses with THz-ABCD system. Both reference (HR Si) and sample (HR Si with $13.6 \mu \mathrm{m} \mathrm{As}_{2} \mathrm{~S}_{3}$ ) are shown. 
The ratio between the spectra recorded for the sample and reference $\tilde{E}_{\text {sam }}(\omega) / \tilde{E}_{\text {ref }}(\omega)$ is then used to determine the amplitude transmission coefficient $\tilde{T}(\omega)$. Knowing the transmission coefficient $\tilde{T}(\omega)$ the complex refractive index $\tilde{n}=n-i \kappa$ can be extracted, where the absorption coefficient $\alpha(\omega)$ is straightforwardly related to the imaginary part of the refractive index by $\alpha=2 \omega \kappa / c$. The refractive index and absorption coefficient of $\mathrm{As}_{2} \mathrm{~S}_{3}$ are shown in Fig. 3.

The $\mathrm{THz}$ spectrum of $\mathrm{As}_{2} \mathrm{~S}_{3}$ reveals several important features which will be discussed below. At lower frequencies ranging from 0.2 to $4 \mathrm{THz}$ monotonous decrease of the refractive index $n$ from 2.74 to 2.67 occurs. Such anomalous dispersion for the $\mathrm{As}_{2} \mathrm{~S}_{3}$ glass (though with slightly higher values from approximately 2.83 to 2.78 ) in the frequency range from 0.5 to $2 \mathrm{THz}$ were also reported by Parrott et al. ${ }^{27}$ The absorption coefficient increases monotonously from 1.2 to $200 \mathrm{~cm}^{-1}$ in the same frequency range. The absorption in this region is expected to follow a universal scaling behavior, ${ }^{28}$ characterized by a power-law frequency dependence of the absorption coefficient. The universality of such a scaling law is, however, limited to frequencies below a point which sometimes is called the Ioffe-Regel transition, ${ }^{29}$ The Ioffe-Regel transition occurs when the mean free path between scattering events of acoustic plane waves propagating in the material approaches the wavelength of the plane wave. At wavelengths longer than this length, scattering will be the dominant loss factor and result in a universal behavior of the absorption coefficient. At shorter wavelengths, plane-wave propagation dominates. Here we directly observe this transition from disorder-induced loss to a collective response mediated by medium- and long-range order of the glass. At intermediate frequencies, ranging from 4.5 to $7 \mathrm{THz}$, the $\mathrm{As}_{2} \mathrm{~S}_{3}$ refractive index displays four pronounced resonances with refractive indices oscillating between 2.6 and 2.7. These dispersion resonances are constant with respect to positions and amplitudes for samples with different thicknesses, as shown in the inset of Fig. 3. The vibrational modes in this frequency range have also been

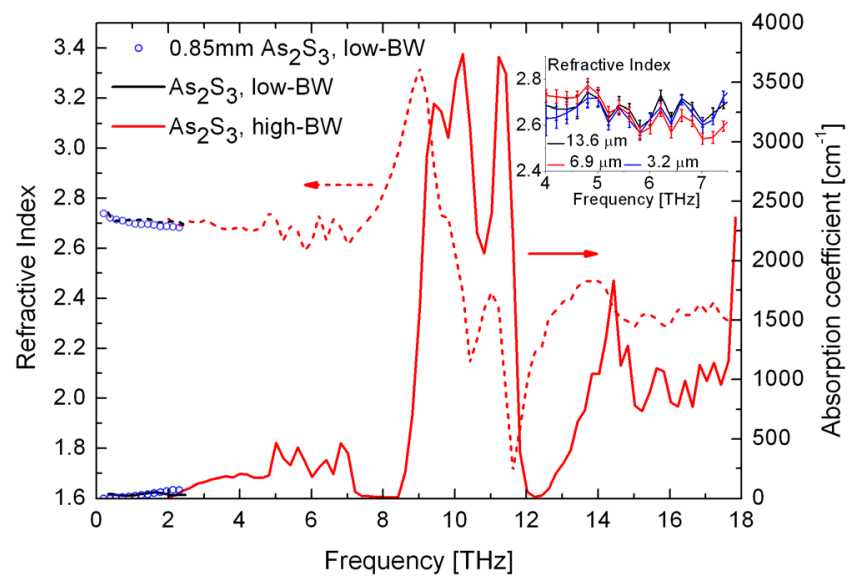

FIG. 3. (Color online) Refractive index (dashed line) and absorption coefficient (solid line) of $\mathrm{As}_{2} \mathrm{~S}_{3}$. The spectroscopy results by THz-TDS system on thin (black) samples and on a $0.85 \mathrm{~mm}$ thick (blue circles) sample. The higher frequencies are measured by THz-ABCD system (red) for $13.6 \mu \mathrm{m}$ thick sample. Upper right corner: Transition from relaxational dynamics to localized vibrational dynamics is shown for three different thicknesses (13.6, 6.9, and $3.2 \mu \mathrm{m})$. Error bars represent standard deviation based on three measurement sets. BW: bandwidth. observed by others. ${ }^{30-32}$ X-ray-absorption spectroscopy and Raman scattering study of $\mathrm{As}_{2} \mathrm{~S}_{3}$ glass have revealed the existence of local order in form of $\mathrm{As}_{4} \mathrm{~S}_{4}$ monomers and pyramidally coordinated $\operatorname{As}\left(\mathrm{S}_{1 / 2}\right)_{3}$ units. ${ }^{31,33}$ The four vibrational modes that are observed at this frequency range are caused by $\mathrm{S}-\mathrm{As}-\mathrm{S}$ bending modes in $\mathrm{As}_{4} \mathrm{~S}_{4}$ monomers. ${ }^{31,32}$

At still higher frequencies the refractive index dispersion exhibits three very strong resonances. The refractive index first increases to 3.3 at $9 \mathrm{THz}$, then abruptly drops to 1.7 at $11.6 \mathrm{THz}$, and finally settles at a value near 2.2 above $14 \mathrm{THz}$. Associated with the strong dispersion are three very strong absorption peaks in the region $8.5-12 \mathrm{THz}$ with the highest absorption coefficient increasing up to $>3700 \mathrm{~cm}^{-1}$. These resonances are local vibrational modes, specifically As-S stretching modes ${ }^{32}$ in both $\mathrm{As}_{4} \mathrm{~S}_{4}$ monomers and pyramidally coordinated $\operatorname{As}\left(\mathrm{S}_{1 / 2}\right)_{3}$ units. The very strong resonances are sandwiched between two transparency windows at 7.2-8.5 and $12-13 \mathrm{THz}$, where the absorption coefficient is measured to be as low as $10 \mathrm{~cm}^{-1}$. This observation indicates that transmission windows exist in $\mathrm{As}_{2} \mathrm{~S}_{3}$, with a bandwidth of several hundred GHz. This, in combination with the high third-order nonlinearity that can be expected in the material, opens up the possibility of compact devices for all-optical processing of ultrahigh-bandwidth signals encoded on $\mathrm{THz}$ waves near 8 and $12 \mathrm{THz}$. We observe good quantitative agreement between the spectra obtained with the two experimental setups on the various $\mathrm{As}_{2} \mathrm{~S}_{3}$ samples.

The GaLaS samples display a high refractive index at low frequencies, as shown in Fig. 4. A refractive index in the range $n \approx 3.5-3.6$ is measured at frequencies from 0.2 to $5 \mathrm{THz}$. For higher frequencies the refractive index decreases dramatically, reaching a minimum value of approximately 1.25 at $11.5 \mathrm{THz}$ and settles a value of 1.7-1.8 at the highest measured frequencies. Following the dispersion trends, GaLaS experiences a very strong absorption band, reaching its maximum of $>6300 \mathrm{~cm}^{-1}$ at $9.8 \mathrm{THz}$. The lowest absorption coefficient reaches approximately $1700 \mathrm{~cm}^{-1}$ at $13 \mathrm{THz}$. Again good matching between the spectra recorded with the two experimental setups on various samples is observed.

In conclusion, we have measured the refractive index and absorption coefficient of $\mathrm{As}_{2} \mathrm{~S}_{3}$ and GaLaS glasses in the

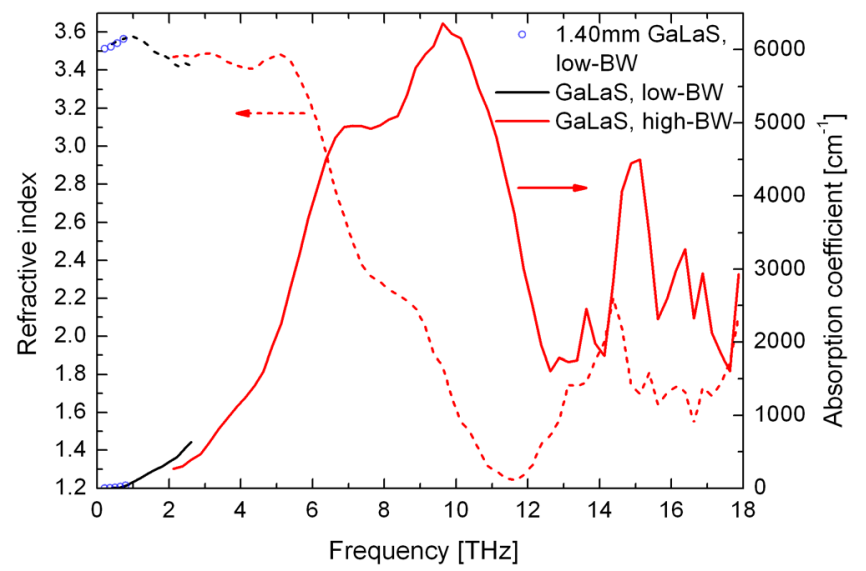

FIG. 4. (Color online) Refractive index (dashed line) and absorption coefficient (solid line) of GaLaS. The spectroscopy results by THz-TDS system on thin (black) samples and on a $1.45 \mathrm{~mm}$ thick (blue circles) sample. The higher frequencies are measured by THz-ABCD system (red) for $3.6 \mu \mathrm{m}$ thick sample. 
broad frequency range extending from 0.2 to $18 \mathrm{THz}$. This broad band frequency range was covered by two different terahertz time-domain spectroscopy setups, and the measured spectra are well matched at the bordering spectral region between the frequency coverage of the two spectrometers. We observe that chalcogenide glasses possess highly complex dispersion profiles. In $\mathrm{As}_{2} \mathrm{~S}_{3}$ we observe the IoffeRegel transition in the $4-5 \mathrm{THz}$ region, followed by a rich vibrational behavior between $4.5-7 \mathrm{THz}$, indicative of medium-range order, and strong, phonon-like vibrational modes in the $8.5-12 \mathrm{THz}$, indicative of local order in the glass. In addition, two transparency windows have been identified in $\mathrm{As}_{2} \mathrm{~S}_{3}$ at frequencies $7.2-8.5$ and $12-13 \mathrm{THz}$. These windows can be of high importance when considering $\mathrm{As}_{2} \mathrm{~S}_{3}$ for future applications in nonlinear $\mathrm{THz}$ devices. The spectroscopy of GaLaS has revealed a high refractive index of 3.5 at lower frequencies, whereas at frequencies higher than $5 \mathrm{THz}$ the refractive index decreases reaching its minimum of 1.25 at $11.5 \mathrm{THz}$. The strong index variations are accompanied by a high absorption over the whole $\mathrm{THz}$ range.

This work has been supported by the Danish Research Council for Technology and Production Sciences through the Project No. 09-070397-FTP, and by the Carlsberg Foundation.

${ }^{1}$ R. Frerichs, J. Opt. Soc. Am. 43, 1153 (1953).

${ }^{2}$ B. J. Eggleton, B. Luther-Davies, and K. Richardson, Nat. Photon. 5, 141 (2011).

${ }^{3}$ A. Zakery and S. R. Elliott, J. Non-Cryst. Solids 330, 1 (2003).

${ }^{4}$ K. Suzuki and T. Baba, Opt. Express 18, 26675 (2010).

${ }^{5}$ T. Han, S. Madden, D. Bulla, and B. Luther-Davies, Opt. Express 18, 19286 (2010).

${ }^{6}$ S. Madden, D.-Y. Choi, D. Bulla, A. Rode, B. Luther-Davies, V. Táeed, M. Pelusi, and B. Eggleton, Opt. Express 15, 14414 (2007).

${ }^{7}$ S. Wong, M. Deubel, F. Pérez-Willard, S. John, G. A. Ozin, M. Wegener, and G. von Freymann, Adv. Mater. 18, 265 (2006).
${ }^{8}$ M. Popescu, A. Velea, A. Lorinczi, M. Zamfirescu, F. Jipa, S. Miclos, A. Popescu, and D. Savastru, Dig. J. Nanomater. Biostruct. 5, 1579 (2010).

${ }^{9}$ A. Popescu, S. Miclos, D. Savastru, R. Savastru, M. Ciobanu, M. Popescu, A. Lorinczi, F. Sava, A. Velea, F. Jipa, and M. Zamfirescu, J. Optoelectron. Adv. Mater. 11, 1874 (2009).

${ }^{10}$ M. Pelusi, V. Táeed, L. Fu, E. Mägi, M. Lamont, S. Madden. D.-Y. Choi, D. Bulla, B. Luther-Davies, and B. Eggleton, IEEE J. Sel. Top. Quantum Electron. 14, 529 (2008).

${ }^{11}$ Z. Yang, M. Fah, K. Reynolds, J. Sexton, M. Riley, M.-L. Anne, B. Bureau, and P. Lucas, Opt. Express 18, 26754 (2010).

${ }^{12}$ B. Quan, X. Xu, H. Yang, Q. Wang, L. Wang, and C. Gu, Appl. Phys. Lett. 94, 123901 (2009).

${ }^{13}$ Y. Shen, T. Lo, P. Taday, B. Cole, W. Tribe, and M. Kemp, Appl. Phys. Lett. 86, 241116 (2005).

${ }^{14}$ J. Xu, K. Plaxco, and J. Allen, Protein Sci. 15, 1175 (2006).

${ }^{15}$ M. Naftaly and R. Miles, J. Appl. Phys. 102, 043517 (2007).

${ }^{16}$ F. Rutz, M. Koch, L. Micele, and G. Portu, Appl. Opt. 45, 8070 (2006).

${ }^{17}$ V. Astley, B. McCracken, R. Mendis, and D. Mittleman, Opt. Lett. 36, $1452(2011)$

${ }^{18}$ S.-Z. Lo and T. Murphy, Opt. Lett. 19, 2921 (2009).

${ }^{19}$ W. Padilla, A. Taylor, C. Highstrete, M. Lee, and R. Averitt, Phys. Rev. Lett. 96, 107401 (2006).

${ }^{20}$ A. Strikwerda, K. Fan, H. Tao, D. Pilon, X. Zhang, and R. Averitt, Opt. Express 17, 136 (2009).

${ }^{21}$ R. Malureanu, P. U. Jepsen, S. Xiao, L. Zhou, D. G. Cooke, A. Andryieuski, and A.V. Lavrinenko, Proc. SPIE 7711, 77110M (2010).

${ }^{22}$ P. U. Jepsen, D. G. Cooke, and M. Koch, Laser Photon. Rev. 5, 124 (2010).

${ }^{23}$ J. Dai, J. Liu, and X.-C. Zhang, IEEE J. Sel. Top. Quantum Electron. 17, 183 (2011).

${ }^{24}$ J. Dai, X. Xie, and X.-C. Zhang, Phys. Rev. Lett. 97, 103903 (2006).

${ }^{25}$ A. Popescu, D. Savastru, S. Miclos, V. Braic, M. Popescu, A. Manea, and A. Kiss, Dig. J. Nanomater. Biostruct. 6, 341 (2011).

${ }^{26}$ L. Duvillaret, F. Garet, and J.-L. Coutaz, IEEE J. Sel. Top. Quantum Electron. 2, 739 (1996).

${ }^{27}$ E. Parrott, J. Zeitler, L. Gladden, S. Taraskin, and S. Elliott, J. Non-Cryst. Solids 335, 1824 (2009).

${ }^{28}$ S. N. Taraskin, S. Simdyankin, S. Elliott, J. Neilson, and T. Lo, Phys. Rev. Lett. 97, 055504 (2006).

${ }^{29}$ A. Ioffe and A. Regel, Prog. Semicond. 4, 237 (1960).

${ }^{30}$ F. Billes, V. Mitsa, I. Fejes, N. Mateleshko, and I. Fejsa, J. Mol. Struct. 513, 109 (1999).

${ }^{31}$ D. Georgiev, P. Boolchand, and K. Jackson, Philos. Mag. 83, 2941 (2003).

${ }^{32}$ H. Whitfield, Aust. J. Chem. 24, 697 (1971).

${ }^{33}$ C. Yang, M. Paesler, and D. Sayers, Phys. Rev. B 39, 10342 (1988). 